\title{
Clinical characteristics, risk factors and outcomes of mixed Candida albicans/ bacterial bloodstream infections
}

Li Zhong $^{1,2+}$, Shufang Zhang ${ }^{3 \dagger}$, Kankai Tang ${ }^{2 \dagger}$, Feifei Zhou ${ }^{1,4}$, Cheng Zheng ${ }^{1,5}$, Kai Zhang ${ }^{1}$, Jiachang Cai ${ }^{6}$, Hongwei Zhou ${ }^{6}$, Yesong Wang ${ }^{1}$, Baoping Tian' ${ }^{1}$, Zhaocai Zhang ${ }^{1}$, Wei Cui ${ }^{1 *}$, Zhaohui Dong $^{2^{*}}$ and Gensheng Zhang ${ }^{1 *}$ (1)

\begin{abstract}
Purpose: The purpose of this study was to explore the clinical features, risk factors, and outcomes of mixed Candida albicans/bacterial bloodstream infections (mixed-CA/B-BSIs) compared with monomicrobial Candida albicans bloodstream infection (mono-CA-BSI) in adult patients in China.

Methods: All hospitalized adults with Candida albicans bloodstream infection (CA-BSI) were recruited for this retrospective observational study from January 1, 2013, to December 31, 2018.

Results: Of the 117 patients with CA-BSI, 24 patients (20.5\%) had mixed-CA/B-BSIs. The most common copathogens were coagulase-negative Staphylococcus (CNS) (24.0\%), followed by Klebsiella pneumoniae (20.0\%) and Staphylococcus aureus (16.0\%). In the multivariable analysis, a prior ICU stay > 2 days (adjusted odds ratio [OR], 7.445; 95\% confidence interval [CI], 1.152-48.132) was an independent risk factor for mixed-CA/B-BSIs. Compared with patients with mono-CA-BSI, patients with mixed-CA/B-BSIs had a prolonged length of mechanical ventilation [17.5 $(4.5,34.8)$ vs. $3.0(0.0,24.5), p=0.019]$ and prolonged length of ICU stay $[22.0(14.3,42.2)$ vs. $8.0(0.0,31.5), p=0.010]$; however, mortality was not significantly different.
\end{abstract}

Conclusions: There was a high rate of mixed-CA/B-BSIs cases among CA-BSI cases, and CNS was the predominant coexisting species. A prior ICU stay $>2$ days was an independent risk factor for mixed -CA/B-BSIs. Although there was no difference in mortality, the outcomes of patients with mixed -CA/B-BSIs, including prolonged length of mechanical ventilation and prolonged length of ICU stay, were worse than those with mono-CA-BSI; this deserves further attention from clinicians.

Keywords: Candidemia, Bloodstream infections, Mixed Candida/bacterial bloodstream infections, Candida albicans, Mortality, Risk factor

\footnotetext{
*Correspondence: zricu@zju.edu.cn; 1409640178@qq.com;

genshengzhang@zju.edu.cn

'Li Zhong, Shufang Zhang and Kankai Tang contributed equally to this work.

'Department of Critical Care Medicine, Second Affiliated Hospital, Zhejiang

University School of Medicine, Hangzhou 310009, Zhejiang, China

${ }^{2}$ Department of Critical Care Medicine, First Affiliated Hospital, Huzhou

Teachers College, the First People's Hospital of Huzhou, Huzhou 313000,

Zhejiang, China

Full list of author information is available at the end of the article
}

(c) The Author(s). 2020 Open Access This article is licensed under a Creative Commons Attribution 4.0 International License, which permits use, sharing, adaptation, distribution and reproduction in any medium or format, as long as you give appropriate credit to the original author(s) and the source, provide a link to the Creative Commons licence, and indicate if changes were made. The images or other third party material in this article are included in the article's Creative Commons licence, unless indicated otherwise in a credit line to the material. If material is not included in the article's Creative Commons licence and your intended use is not permitted by statutory regulation or exceeds the permitted use, you will need to obtain permission directly from the copyright holder. To view a copy of this licence, visit http://creativecommons.org/licenses/by/4.0/. The Creative Commons Public Domain Dedication waiver (http://creativecommons.org/publicdomain/zero/1.0/) applies to the data made available in this article, unless otherwise stated in a credit line to the data. 


\section{Introduction}

In critically ill patients, bloodstream infection (BSI) is an important cause of morbidity and mortality. Candidemia is a leading cause of healthcare-associated BSI, with allcause in-hospital mortality reaching $30 \%$ in the United States [1-3]. With the extensive use of antibiotics and immunosuppressants and rapid increases in the applications of invasive medical examinations and treatments, Candida has gradually become a significant pathogen responsible for BSI, with a crude average incidence of 8.7 per 100,000 population [2]. Patients with candidemia have many typical risk factors, including recent surgery, use of broad-spectrum antibiotics, presence of a central venous catheter (CVC), and injection drug use [2].

Although many candidemia cases are monomicrobial, mixed Candida/bacterial BSIs account for $18-56 \%$, as previously reported [4-8]. In these studies $[4-6,8]$, the following limitations existed: (1) Although the clinical significance and prognosis of mixed Candida/bacterial BSIs versus monomicrobial candidemia were investigated, few reports focused on specific Candida species, such as C. albicans. (2) A recent study reported that patients with mixed Candida/bacterial BSIs had a worse prognosis than patients with monomicrobial candidemia (45.0\% crude mortality vs. $17.8 \%$ crude mortality, $p<$ 0.05 ) [8], while other studies did not observe a similar mortality rate [4-6]. The discrepancies in this clinical outcome among different studies are not understood. (3) Some risk factors associated with mixed Candida/bacterial BSIs, such as prolonged length of prior hospital stay ( $\geq 7$ weeks), septic shock at the time of candidemia, a high acute physiology chronic health evaluation (APACHE) II score, and use of antibiotics, are supported by data mainly from Korea and Spain. Whether these risk factors also apply in other countries, such as China, is unknown. Therefore, it is necessary to investigate the clinical features of mixed Candida/bacterial BSIs involving specific Candida species in China.

Although an increase in the proportion of non-albicans Candida species was observed in some epidemiologic studies [9, 10], C. albicans is still the most common species isolated from patients with candidemia, followed by C. glabrata and C. parapsilosis [2-4, $10,11]$. The following differences were found among different Candida spp. (1) Different distributions were observed among different Candida spp. C. albicans is the predominant species associated with ICU-related infections, while C. glabrata is most commonly associated with gastrointestinal tract diseases [11]. (2) Different resistance rates to common antifungal agents have been observed between C. albicans and non-albicans Candida species. Non-albicans Candida species are more likely to be resistant to fluconazole than $C$. albicans [11]. (3) Different outcomes, such as mortality, also exist between non-albicans candidemia and $C$. albicans infection $[9,10]$. Whether there are differences in the sensitivity to antifungal agents, the severity of illness or mortality between those with mixed-CA/B-BSIs and mono-CA-BSI, and which factors are associated with mixed-CA/B-BSIs are not well known. Given that $C$. albicans is the most common species responsible for candidemia and few reports about mixed-candidemia involving a specific Candida species, such as $C$. albicans, exist, we performed the study to investigate the clinical characteristics of, risk factors for and outcomes of mixed-CA/B-BSIs compared with mono-CA-BSI.

\section{Material and methods}

\section{Patients and study design}

From January 2013 to December 2018, we conducted a single-center retrospective cohort study at the Second Affiliated Hospital of Zhejiang University School of Medicine, a teaching hospital with 3200 beds in Hangzhou, China. The study was approved by the Human Ethics Board of the Ethics Committee of the Second Affiliated Hospital of Zhejiang University Medical College. Due to its retrospective nature, the Ethics Committee determined that patient consent was not required.

Patients positive for C. albicans according to blood culture were recruited. Candidemia that occurred 30 days after the initial episode was considered a new case [10]. The exclusion criteria were as follows: a) age $<18$ years old; b) incomplete or missing case data; and c) the presence of nonpathogenic C. albicans. Common skin flora (e.g., Bacillus spp., Corynebacterium spp., Micrococcus spp., Streptococci, Lactobacillus spp. and CNS) were considered pathogenic only when they were present in two or more consecutive blood cultures from at least two separate blood draws or from two separate sites on the same or two consecutive calendar days. Moreover, at the time of specimen collection, the patients must have at least one of the following signs or symptoms: fever $\left(>38.0^{\circ} \mathrm{C}\right)$, chills, or hypotension [12, 13]. Thus, a total of 147 blood culture specimens containing $C$. albicans were initially screened, and a final total of 117 cases were recruited, with 24 cases of mixed-CA/B-BSIs and 93 cases of mono-CA-BSI (Fig. 1).

\section{Data collection}

Patient data were collected from electronic medical records. Patient characteristics included age, sex, severity of illness, sequential organ failure assessment (SOFA) score, and APACHE II score in the first $24 \mathrm{~h}$ following candidemia onset. Data regarding prior ICU stay, prior hospital stay, underlying diseases, immune status, hospitalization ward, life-sustaining treatments $\geq 24 \mathrm{~h}$, 


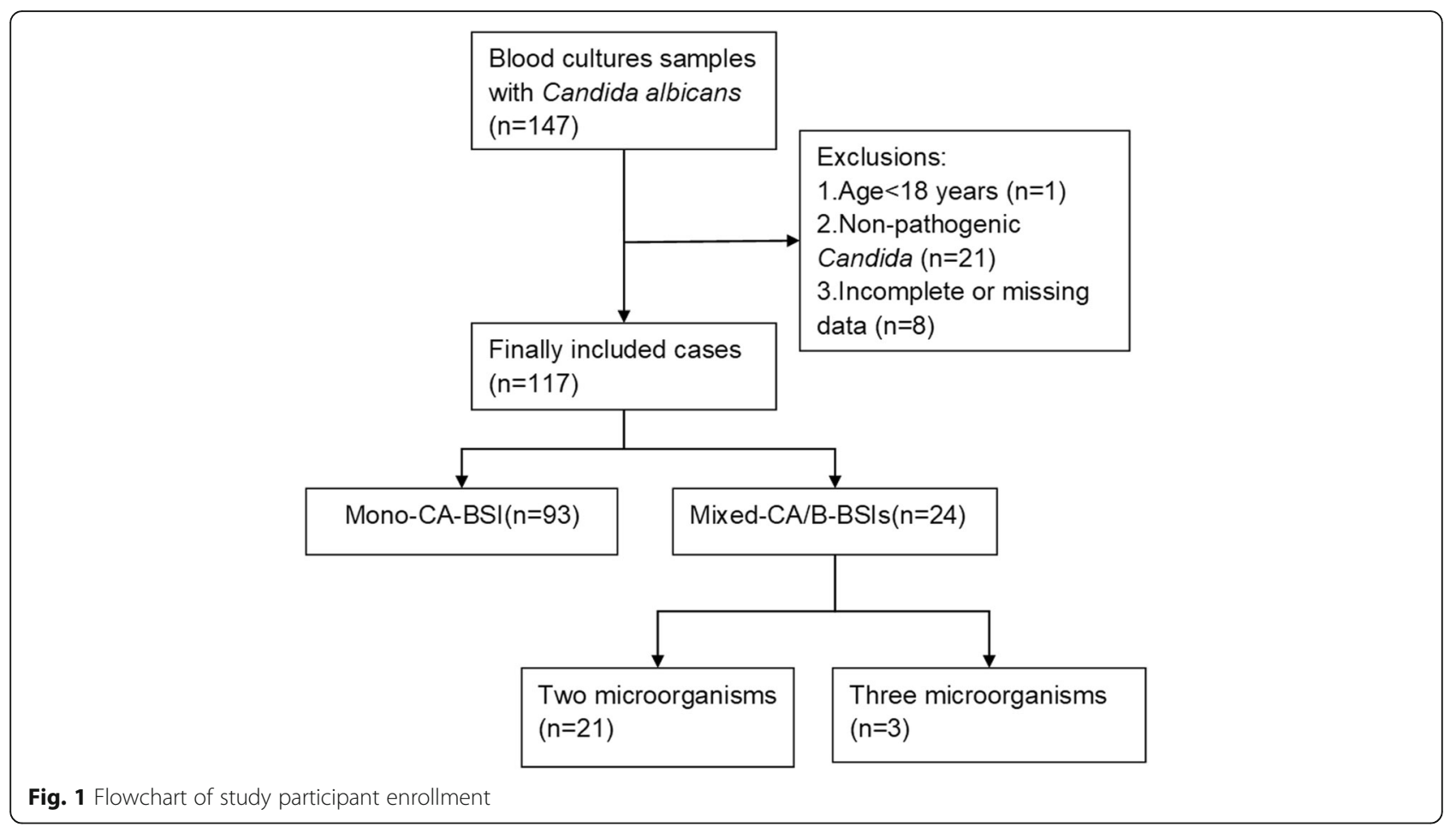

prior use of antibiotics or antifungal agents, previous treatments such as surgical procedures, source control methods and outcomes (length of hospital stay, length of ICU stay, septic shock after the onset of BSI and 28-day mortality after the onset of BSI) were collected. Microbiological data, such as copathogens in mixed-CA/BBSIs, likely sources of BSI and sensitivity to antimycotics, were also recorded.

\section{Species identification and antimycotic sensitivity test}

Blood samples were collected following rigorous skin disinfection to obtain at least two sets of aerobic and anaerobic blood cultures (10-20 mL per bottle) when the patients were suspected of BSI with clinical manifestations, i.e., fever $>38.0^{\circ} \mathrm{C}$, chills, hypotension, low-grade fever at $38{ }^{\circ} \mathrm{C}$ or even hypothermia if sepsis was suspected [12]. Species identification of both bacteria and yeasts was performed by matrix-assisted laser desorption/ionization time of flight mass spectrometry (MALDI-TOF MS) (Bruker Daltonik GmbH, Bremen, Germany). Antimicrobial susceptibility testing for bacteria and yeasts was carried out with a Vitek 2 Compact system and ATB FUNGUS 3 panel (bioMerieux, France), respectively. The results for bacteria and $C$. albicans were interpreted according to breakpoints defined by the Clinical Laboratory Standards Institute $[14,15]$. Because echinocandins were not included in the ATB FUNGUS 3 panel, the results of caspofungin susceptibility were unknown.

\section{Definitions}

Candidemia was defined as the isolation of Candida in blood culture accompanied by fever, chills or hypotension and other corresponding clinical symptoms and signs and the exclusion of specimen contamination [16]. If the identified Candida species was C. albicans, CA-BSI was considered. Mixed-CA/B-BSIs were defined as the isolation of a bacterial organism from blood cultures obtained within $48 \mathrm{~h}$ before or after the onset of CA-BSI [9]. An infection was considered a healthcareassociated infection (HAI) if the date of the event (specific infection criteria) occurred on or after the 3rd calendar day of admission in an inpatient department; the day of admission to the inpatient department was regarded as calendar day 1 [13]. A definitive diagnosis of catheter-related bloodstream infection (CRBSI) required that the same organism was cultivated from at least one percutaneous blood culture and catheter tip culture or that two cultured blood samples (one from a catheter hub and the other from a peripheral vein) met the CRBSI criteria for quantitative blood culture or differential time to positivity [17]. If no other primary source of infection for candidemia can be assigned as secondary is found, a primary BSI with Candida is identified [12]. The timing of administration of antifungal therapy was defined as the interval between the time at which the first $C$. albicans-positive blood sample for culture was drawn and the time at which antifungal treatment was first administered [18]. Antifungal therapy was considered appropriate if the isolated Candida spp. was 
sensitive to the chosen antifungal agent and the antifungal agent was administered with an adequate dosage (for example, fluconazole was administered with a loading dose of $800 \mathrm{mg}$ [12 mg/kg] followed by $400 \mathrm{mg}$ [6 mg/kg] daily, and caspofungin was administered with a loading dose of $70 \mathrm{mg}$ followed by $50 \mathrm{mg}$ daily) [3, 19]. A delay in empiric antifungal treatment was considered when initial administration occurred more than $12 \mathrm{~h}$ after the first positive blood sample [18]. Appropriate antibiotic therapy was defined as antibiotic therapy for bacteremia, where applicable, and sensitivity of the pathogen to the agent [20]. Septic shock was consistent with the Third International Consensus Definitions for Sepsis and Septic Shock (Sepsis-3) [21].

\section{Statistical analyses}

Statistical analysis was performed with SPSS 20.0 software (IBM Corp, Armonk, NY, USA). Continuous variables are presented as the means \pm standard deviations if the data were normally distributed and as medians and interquartile ranges (IQRs) if the data were nonnormally distributed. Continuous variables were compared by Student's t-test or the Mann-Whitney U test, and enumerated variables were compared by the Pearson $x^{2}$ or Fisher's exact test, where appropriate. Variables with $p$-values $<0.05$ in the univariate analysis were entered into the multivariable model. Continuous variables were treated as dichotomous variables based on the Youden index. The multivariate analysis was performed with logistic regression to identify independent risk factors for mixed-CA/B-BSIs. Kaplan-Meier survival estimates were used to generate the survival curves. Differences between survival curves were assessed with log-rank tests. A two-tailed $p<0.05$ was considered statistically significant.

\section{Results}

\section{Demographics and clinical characteristics}

The median age was 68 years (IQR, $59-75$ years), and $58.1 \%(68 / 117)$ of the patients were male. Mono-CABSI and mixed-CA/B-BSIs were responsible for 93/ 117 (79.5\%) and 24/117 (20.5\%) cases, respectively. The most common ward associated with CA-BSI occurrence was the ICU (66.7\%), followed by the surgical ward $(23.9 \%)$ and medical ward $(9.4 \%)$. Solid tumors were the most common comorbidity (28.2\%), followed by diabetes mellitus $(23.9 \%)$. There were no significant differences in age, sex, immune status, or illness severity between the two groups. In the surgical patients and ICU patients, 65.8\% (77/117) and $66.7 \%$ (78/117) episodes were documented, respectively. Other common predisposing factors for candidemia included CVC insertion (106/117, 90.6\%), urethral catheter insertion (106/117, 90.6\%), prior antibiotic exposure $(93 / 117,79.5 \%)$ and total parenteral nutrition (TPN) (85/117, 72.6\%). Compared with the mono-CA-BSI group, the mixed-CA/B-BSIs group had a longer ICU stay before candidemia onset $[12.0(8.0,17.8)$ vs. $1.0(0.0,11.0)$ days, $p=0.001]$, longer hospital stay before candidemia onset [19.0 $(12.0,30.8)$ vs. $12.0(2.0,26.5)$ days, $p=0.031]$, longer duration of mechanical ventilation before candidemia onset $\left[\begin{array}{ll}11.0 & (0.3,24.5) \\ 0.03 & 1.0(0.0,10.0)\end{array}\right.$ days, $p=$ $0.013]$, and longer prior antibiotic exposure before candidemia onset $[17.0(10.3,28.8)$ vs. $8.0(1.0,20.5)$ days, $p=0.007]$; additionally, they were more likely to have an indwelling hemodialysis catheter $[41.7 \%$ vs. $18.3 \%, p=0.015]$ and presence of two or more central venous catheters $[50.0 \%$ vs. $25.8 \%, p=0.022]$, and they had higher rates of life-sustaining treatments such as invasive mechanical ventilation $(81.8 \%$ vs. $54.7 \%, p=0.020)$ and continuous renal replacement therapy (CRRT) $(41.7 \%$ vs. $21.5 \%, p=0.044)$. Nonetheless, there were no significant differences in the proportions of surgical patients, blood transfusion, TPN, or hypoproteinemia (see Table 1). The main source of CA-BSI was CVCs $(29.1 \%, 34 / 117)$, followed by intra-abdominal catheters (20.5\%, 24/ 117). The main sources of mixed-CA/B-BSIs were CVCs $(29.2 \%, 7 / 24)$ and primary sources $(29.2 \%, 7 /$ 24). The main source of mono-CA-BSI was CVCs $(29.0 \%, 27 / 93)$, followed by intra-abdominal catheters $(19.4 \%, 18 / 93)$. Compared with the sources of Candida in mono-CA-BSI, the sources of Candida in mixed-CA/B-BSIs were not significantly different, as shown in Table 2. Regarding infection source control, the rate of CVC removal within $48 \mathrm{~h}$ after the first positive sample in the mixed CA/B-BSI group was higher than that in the mono-CA-BSI group $(54.2 \%$ vs. $29.0 \%$, $p=0.021$ ), but there was no significant difference in the rate of fungal collection from drainage fluid between the two groups ( $20.8 \%$ vs. $15.1 \%, p=0.708)$ (see Table 2$)$.

A high rate of delay of initiation of empiric antifungal treatment (85.5\%) was observed among all patients, and no difference was found between the mixed-CA/B-BSIs $(75.0 \%)$ and mono-CA-BSI (88.2\%) groups. In addition, the total rate of appropriate antifungal therapy was less than $50 \%(36.8 \%)$, and it was similar between patients with mixed-CA/B-BSIs (33.3\%) and those with monoCA-BSI (37.6\%), as shown in Table 2. The proportions of empiric treatment and appropriate antibiotic therapy for bacteremia in mixed-CA/B-BSIs patients were $33 \%$ $(8 / 24)$ and $70 \%(17 / 24)$, respectively.

\section{Antifungal susceptibility}

The isolated C. albicans in both groups exhibited 100\% susceptibility to amphotericin B, voriconazole, and no resistance to fluconazole was observed. Notably, in the 
Table 1 Baseline characteristics of the patients with mono-CA-BSI or mixed-CA/B-BSIs

\begin{tabular}{|c|c|c|c|c|}
\hline Characteristics & $\operatorname{Total}(n=117)$ & Mono-CA-BSI $(n=93)$ & Mixed-CA/B-BSIs $(n=24)$ & Pvalue \\
\hline Age, median years (IQR) & $68(59,75)$ & $69(59,76)$ & $64(47,74)$ & 0.399 \\
\hline Male sex [n (\%)] & $68(58.1 \%)$ & $53(56.9 \%)$ & $15(62.5 \%)$ & 0.626 \\
\hline APACHE II score at the onset of candidemia (IQR) & $17.0(11.5,24.5)$ & $17.0(12.0,24.0)$ & $17.5(10.0,26.5)$ & 0.863 \\
\hline SOFA score at the onset of candidemia (IQR) & $6.0(2.0,9.0)$ & $5.0(2.0,9.0)$ & $6.5(2.0,9.8)$ & 0.494 \\
\hline Prior ICU stay (days) (IQR) & $3.0(0.0,14.0)$ & $1.0(0.0,11.0)$ & $12.0(8.0,17.8)$ & 0.001 \\
\hline Prior hospital stay (days) (IQR) & $14.0(4.5,27.5)$ & $12.0(2.0,26.5)$ & $19.0(12.0,30.8)$ & 0.031 \\
\hline Prior ventilation mechanical ventilation (days) (IQR) & $1.0(0.0,13.0)$ & $1.0(0.0,10.0)$ & $11.0(0.3,24.5)$ & 0.013 \\
\hline \multicolumn{5}{|l|}{ Underlying disease [n (\%)] } \\
\hline Diabetes mellitus & $28(23.9 \%)$ & $23(24.7 \%)$ & $5(20.8 \%)$ & 0.690 \\
\hline Chronic cardiac dysfunction & $24(20.5 \%)$ & $16(17.2 \%)$ & $8(33.3 \%)$ & 0.144 \\
\hline Chronic obstructive pulmonary disease & $5(4.3 \%)$ & $5(5.4 \%)$ & $0(0 \%)$ & 0.552 \\
\hline Chronic renal insufficiency & $9(7.7 \%)$ & $8(8.6 \%)$ & $1(4.2 \%)$ & 0.766 \\
\hline Chronic hepatic insufficiency & $14(12.0 \%)$ & $13(14.0 \%)$ & $1(4.2 \%)$ & 0.333 \\
\hline Solid tumour & $33(28.2 \%)$ & $28(30.1 \%)$ & $5(20.8 \%)$ & 0.368 \\
\hline Haematological malignancy & $1(0.9 \%)$ & $1(1.1 \%)$ & $0(0 \%)$ & $>0.999$ \\
\hline Trauma & $19(16.2 \%)$ & $14(15.1 \%)$ & $5(20.8 \%)$ & 0.708 \\
\hline Burn injury & $4(3.4 \%)$ & $2(2.2 \%)$ & $2(8.3 \%)$ & 0.186 \\
\hline Transplant & $14(12.0 \%)$ & $11(11.8 \%)$ & $3(12.5 \%)$ & $>0.999$ \\
\hline \multicolumn{5}{|l|}{ Immunocompromised [n (\%)] } \\
\hline Immunosuppressant therapy & $6(5.1 \%)$ & $6(6.5 \%)$ & $0(0.0 \%)$ & 0.448 \\
\hline Steroid therapy & $6(5.1 \%)$ & $6(6.5 \%)$ & $0(0.0 \%)$ & 0.448 \\
\hline Chemotherapy/radiation & $7(6.0 \%)$ & $7(7.5 \%)$ & $0(0.0 \%)$ & 0.366 \\
\hline Neutropenia & $4(3.4 \%)$ & $3(3.2 \%)$ & $1(4.2 \%)$ & $>0.999$ \\
\hline Blood transfusion [n (\%)] & $40(34.2 \%)$ & $30(32.2 \%)$ & $10(41.7 \%)$ & 0.386 \\
\hline \multicolumn{5}{|l|}{ Hospitalization ward [n (\%)] } \\
\hline Medical & $11(9.4 \%)$ & $11(11.8 \%)$ & $0(0.0 \%)$ & 0.168 \\
\hline Surgical & $28(23.9 \%)$ & $24(25.8 \%)$ & $4(16.7 \%)$ & 0.349 \\
\hline ICU & $78(66.7 \%)$ & $58(62.4 \%)$ & $20(83.3 \%)$ & 0.052 \\
\hline Nosocomial infection [n (\%)] & $112(95.7 \%)$ & $88(94.6 \%)$ & $24(100 \%)$ & 0.552 \\
\hline \multicolumn{5}{|l|}{ Life-sustaining treatments $\geq 24 \mathrm{~h}$ [n (\%)] } \\
\hline Invasive mechanical ventilation & $65(60.2 \%)$ & $47(54.7 \%)$ & $18(81.8 \%)$ & 0.020 \\
\hline Vasopressor & $45(38.5 \%)$ & $34(36.6 \%)$ & $11(45.8 \%)$ & 0.405 \\
\hline CRRT & $30(25.6 \%)$ & $20(21.5 \%)$ & $10(41.7 \%)$ & 0.044 \\
\hline \multicolumn{5}{|l|}{ Catheterisation ${ }^{a}[\mathrm{n}(\%)]$} \\
\hline Central venous catheter ${ }^{\mathrm{b}}$ & $106(90.6 \%)$ & $83(89.2 \%)$ & $23(95.8 \%)$ & 0.553 \\
\hline Hemodialysis catheter ${ }^{c}$ & $27(23.1 \%)$ & $17(18.3 \%)$ & $10(41.7 \%)$ & 0.015 \\
\hline PICC & $13(11.1 \%)$ & $10(10.8 \%)$ & $3(12.5 \%)$ & $>0.999$ \\
\hline Peripheral arterial catheters & $37(31.6 \%)$ & $29(31.2 \%)$ & $8(33.3 \%)$ & 0.840 \\
\hline Drainage tube & $77(65.8 \%)$ & $59(63.4 \%)$ & $18(75.0 \%)$ & 0.287 \\
\hline Urethral catheter & $106(90.6 \%)$ & $85(91.4 \%)$ & $21(87.5 \%)$ & 0.848 \\
\hline Presence of two or more central venous catheters & $36(30.8 \%)$ & $24(25.8 \%)$ & $12(50.0 \%)$ & 0.022 \\
\hline Total parenteral nutrition [n (\%)] & $85(72.6 \%)$ & $65(69.9 \%)$ & $20(83.3 \%)$ & 0.188 \\
\hline Hypoproteinemia [n (\%)] & $49(41.9 \%)$ & $37(39.8 \%)$ & $12(50.0 \%)$ & 0.366 \\
\hline Surgery [n (\%)] & $77(65.8 \%)$ & $59(63.4 \%)$ & $18(75.0 \%)$ & 0.287 \\
\hline Abdominal & $39(33.3 \%)$ & $32(34.4 \%)$ & $7(29.2 \%)$ & 0.627 \\
\hline
\end{tabular}

Notes: Bold, indicates $P<0.05$

Abbreviations: IQR interquartile range, COPD chronic obstructive pulmonary disorder, SOFA sequential organ failure assessment, $A P A C H E$ acute physiology and chronic health evaluation, ICU intensive care unit, CRRT continuous renal replacement therapy, PICC Peripherally inserted central catheters ${ }^{a}$ Included patients who were required to be catheterised within 2 weeks of the first positive sample, regardless of whether or not the catheter was removed before diagnosis

${ }^{\mathrm{b}}$ Non-tunneled central venous catheters such as subclavian, internal jugular and femoral venous catheters excluding hemodialysis catheter and PICC

'Non-tunneled temporary dialysis catheter 
Table 2 The source of candidemia, prior antibiotic and antifungal therapy of the mono-CA-BSI compared with the Mixed-CA/B-BSIs

\begin{tabular}{|c|c|c|c|c|}
\hline Variable & Total $(n=117)$ & Mono-CA-BSI $(n=93)$ & Mixed-CA/B-BSIs $(n=24)$ & $P$ value \\
\hline \multicolumn{5}{|l|}{ Source of candidaemia [n (\%)] } \\
\hline Definitive CVC-related & $34(29.1 \%)$ & $27(29.0 \%)$ & 7 (29.2\%) & 0.990 \\
\hline Intra-abdominal & $24(20.5 \%)$ & $18(19.4 \%)$ & $6(25.0 \%)$ & 0.744 \\
\hline Primary & $22(18.8 \%)$ & $15(16.1 \%)$ & $7(29.2 \%)$ & 0.244 \\
\hline Lower respiratory tract & $12(10.3 \%)$ & $11(11.8 \%)$ & $1(4.2 \%)$ & 0.468 \\
\hline Urinary tract infection & $7(6.0 \%)$ & $6(6.5 \%)$ & $1(4.2 \%)$ & $>0.999$ \\
\hline Gastrointestinal tract & $6(5.1 \%)$ & $6(6.5 \%)$ & $0(0.0 \%)$ & 0.344 \\
\hline Skin and Soft tissue & $5(4.3 \%)$ & $4(4.3 \%)$ & $1(4.2 \%)$ & $>0.999$ \\
\hline Meningitis & $3(2.6 \%)$ & $2(2.2 \%)$ & $1(4.2 \%)$ & 0.501 \\
\hline Endocardium & $2(1.7 \%)$ & $2(2.2 \%)$ & $0(0.0 \%)$ & $>0.999$ \\
\hline Osteoarthritis & $1(0.9 \%)$ & $1(1.1 \%)$ & $0(0.0 \%)$ & $>0.999$ \\
\hline \multicolumn{5}{|l|}{ Source control [n (\%)] } \\
\hline Removal of contaminated lines ${ }^{a}$ & $40(34.2 \%)$ & $27(29.0 \%)$ & $13(54.2 \%)$ & 0.021 \\
\hline Draining of fungal collection & $19(16.2 \%)$ & $14(15.1 \%)$ & $5(20.8 \%)$ & 0.708 \\
\hline Days of prior antibiotic exposure (IQR) & $11.0(3.0,22.0)$ & $8.0(1.0,20.5)$ & $17.0(10.3,28.8)$ & 0.007 \\
\hline Prior antibiotic exposure ${ }^{\mathrm{b}}[\mathrm{n}(\%)]$ & $93(79.5 \%)$ & $69(74.2 \%)$ & $24(100.0 \%)$ & 0.012 \\
\hline Cephalosporins & $33(28.2 \%)$ & $25(26.9 \%)$ & $8(33.3 \%)$ & 0.531 \\
\hline Carbapenems & 49 (41.9\%) & $41(44.1 \%)$ & $8(33.3 \%)$ & 0.341 \\
\hline Penicillins & $25(21.4 \%)$ & 19 (20.4\%) & $6(25.0 \%)$ & 0.626 \\
\hline Quinolones & $4(3.4 \%)$ & $4(4.3 \%)$ & $0(0.0 \%)$ & 0.580 \\
\hline \multicolumn{5}{|l|}{ Initial antifungal agent [n (\%)] } \\
\hline Fluconazole & $40(34.2 \%)$ & $32(34.4 \%)$ & $8(33.3 \%)$ & 0.921 \\
\hline Echinocandin & $46(39.3 \%)$ & $36(38.7 \%)$ & $10(41.7 \%)$ & 0.791 \\
\hline Voriconazole & $11(9.4 \%)$ & $9(9.7 \%)$ & $2(8.3 \%)$ & $>0.999$ \\
\hline Prior antifungal exposure [n (\%)] & $10(8.5 \%)$ & $6(6.4 \%)$ & $4(16.7 \%)$ & 0.235 \\
\hline Appropriate Antifungal therapy ${ }^{\complement}[n(\%)]$ & $43(36.8 \%)$ & $35(37.6 \%)$ & $8(33.3 \%)$ & 0.697 \\
\hline Delay in initiation of empiric antifungal treatment ${ }^{\mathrm{d}}[\mathrm{n}(\%)]$ & 100 (85.5\%) & 82 (88.2\%) & $18(75.0 \%)$ & 0.103 \\
\hline
\end{tabular}

Abbreviations: CVC central venous catheter, PICC Peripherally inserted central catheters, CRBSI catheter-related bloodstream infection;

${ }^{a}$ Central venous catheter removed within $48 \mathrm{~h}$ after the first positive sample

${ }^{\mathrm{b}}$ All patients receiving systemic drug therapy for $\geq 3$ days within 2 weeks prior to candidaemia onset

${ }^{C}$ Antifungal therapy was defined as appropriate if the isolated Candida spp. was sensitive to the chosen antifungal agent, and the antifungal agent was used with

adequate dosages (like Fluconazole: loading dose of $800 \mathrm{mg}$ [12 mg/kg], then $400 \mathrm{mg}[6 \mathrm{mg} / \mathrm{kg}$ ] daily; Caspofungin: loading dose of $70 \mathrm{mg}$, then $50 \mathrm{mg}$ daily)

${ }^{\mathrm{d}}$ The delay of empiric antifungal treatment was considered as initial use more than $12 \mathrm{~h}$ after the report of first positive blood sample

mono-CA-BSI and mixed CA/B-BSIs groups, 11 (24.4\%) and $2(13.3 \%)$ cases were completely resistant to ketoconazole, respectively. There was no significant difference between the two groups in the in-vitro antifungal susceptibility test results, as shown in Table 3 . Because the drug sensitivity kit used in our current microbiology laboratory does not include echinocandins, the specific drug sensitivity of $C$. albicans to echinocandins was unclear.

\section{Independent risk factors for mixed-CA/B-BSIs}

Variables with $p$-value of $<0.05$, including a prior hospital stay $>7$ days, a prior ICU stay $>2$ days, prior antibiotic exposure $>7$ days, prior mechanical ventilation $>2$ days, an indwelled hemodialysis catheter and the presence of two or more CVCs at the time of onset of candidemia, were entered into the multivariable logistic regression model to identify factors associated with mixed-CA/B-BSIs. As shown in Table 4, the only independent risk factor for mixed-CA/B-BSIs was a prior ICU stay $>2$ days (adjusted odds ratio [OR], 7.445; 95\% confidence interval [CI], 1.152-48.132).

\section{Species distributions of concomitant bacteria isolated from the mixed-CA/B-BSIs}

The most common copathogens were gram-positive bacteria (52.0\%), followed by gram-negative bacteria (48.0\%). In terms of the exact microorganisms, the most frequent pathogen was CNS (24.0\%), followed by Klebsiella pneumoniae (K. pneumoniae) (20.0\%) and 
Table 3 In vitro antifungal susceptibility of C. albicans between mono-CA-BSI and mixed-CA/B-BSIs

\begin{tabular}{|c|c|c|c|c|c|c|c|c|c|c|}
\hline \multirow{3}{*}{$\begin{array}{l}\text { Candida } \\
\text { species }\end{array}$} & \multirow[t]{3}{*}{ Antifungalagent } & \multicolumn{4}{|c|}{ mono-CA-BSI $(n=93)$} & \multicolumn{4}{|c|}{ mixed-CA/B-BSIs $(n=24)$} & \multirow{3}{*}{$\begin{array}{l}P \\
\text { value }\end{array}$} \\
\hline & & \multirow{2}{*}{$\begin{array}{l}\text { Number } \\
\text { of } \\
\text { strains }\end{array}$} & \multicolumn{3}{|c|}{ Drug sensitivity } & \multirow{2}{*}{$\begin{array}{l}\text { Number } \\
\text { of } \\
\text { strains }\end{array}$} & \multicolumn{3}{|c|}{ Drug sensitivity } & \\
\hline & & & $S$ & 1 & $\mathbf{R}$ & & $S$ & I & $\mathbf{R}$ & \\
\hline \multirow[t]{8}{*}{ C.albicans } & Fluconazole $(n=104)^{a}$ & $85(91.3 \%)$ & $81(95.3 \%)$ & $4(4.7 \%)$ & 0 & 19(79.1\%) & 19(100.0\%) & 0 & 0 & $>0.999$ \\
\hline & Clotrimazole $(n=69)^{\text {a }}$ & $55(59.1 \%)$ & $54(98.2 \%)$ & 0 & $1(1.8 \%)$ & $15(62.5 \%)$ & 14(93.3\%) & 0 & 0 & 0.901 \\
\hline & $\operatorname{Ketoconazole}(n=60)^{a}$ & $45(48.3 \%)$ & $19(42.2 \%)$ & 15(33.3\%) & $11(24.4 \%)$ & $15(62.5 \%)$ & $7(46.7 \%)$ & $6(40.0 \%)$ & $2(13.3 \%)$ & 0.764 \\
\hline & Itraconazole $(n=111)^{\mathrm{a}}$ & $89(95.7 \%)$ & $86(96.6 \%)$ & $1(1.1 \%)$ & $2(2.2 \%)$ & $22(91.7 \%)$ & $21(95.5 \%)$ & $1(4.5 \%)$ & 0 & $>0.999$ \\
\hline & Amphotericin $B(n=111)^{a}$ & $90(96.8 \%)$ & $90(100.0 \%)$ & 0 & 0 & $21(87.5 \%)$ & $21(100.0 \%)$ & 0 & 0 & $>0.999$ \\
\hline & $\operatorname{Nystatin}(n=68)^{a}$ & $56(60.2 \%)$ & $55(98.2 \%)$ & $1(1.8 \%)$ & 0 & $12(50.0 \%)$ & $12(100.0 \%)$ & 0 & 0 & $>0.999$ \\
\hline & 5 -fluorocytosine $(n=38)^{a}$ & $31(33.3 \%)$ & $30(96.8 \%)$ & 0 & $1(3.2 \%)$ & $7(29.1 \%)$ & $7(100.0 \%)$ & 0 & 0 & $>0.999$ \\
\hline & $\operatorname{Voriconazole}(n=103)^{\mathrm{a}}$ & $82(88.2 \%)$ & $82(100.0 \%)$ & 0 & 0 & $21(87.5 \%)$ & $21(100.0 \%)$ & 0 & 0 & $>0.999$ \\
\hline
\end{tabular}

$S$ sensitive, $I$ intermediary, $R$ resistant

${ }^{a}$ Not all agents listed tested in all isolates

Staphylococcus aureus (S. aureus) (16.0\%). The detailed distribution of concomitant bacterial species in mixedCA/B-BSIs is shown in Fig. 2.

\section{Outcomes}

The median length of ICU stay was 14 days (IQR, 1.033.0), and the median length of hospital stay was 33 days (IQR, 18.0-56.0). Compared with patients with monoCA-BSI, patients with mixed -CA/B-BSIs had a prolonged length of ICU stay $[8.0(0.0,31.5)$ vs. 22.0 (14.3, 42.2) days, $p=0.010]$ and longer mechanical ventilation time $[3.0(0.0,24.5)$ vs. $17.5(4.5,34.8)$ days, $p=0.019]$. The incidence of septic shock, 28-day and 60-day mortality, and in-hospital mortality in patients with mixedCA/B-BSIs were not different from those in patients with mono-CA-BSI (Table 5, Fig. 3).

\section{Discussion}

Polymicrobial bacteremia has been reported in previous studies, which was observed in 23.5 and $48.0 \%$ of patients with Acinetobacter baumannii bacteremia and $K$. pneumoniae bacteremia, respectively [22, 23]. In terms of enterococcal BSIs, $34.8 \%$ of cases (157/
451) had coinfection with other pathogens, such as CNS, A. baumannii, or K. pneumoniae [24]. The current report found that the incidence of mixedCA/B-BSIs was $20.5 \%$. A similar proportion of mixed-CA/B-BSIs among CA-BSIs was reported in developed regions of Europe, such as Spain (18\%) [4], Asia, such as South Korea (23\%) [5], and China (20\%) [8]. These results suggest that relatively high proportions of specific polymicrobial BSIs are observed not only in bacterial BSIs but also in candidemia and CA-BSIs.

Similar risk factors were found to be associated with mixed-CA/B-BSIs in previous studies $[4,5]$, including a prolonged ICU stay, a prolonged hospital stay before candidemia onset, antimicrobial administration, the presence of an indwelling hemodialysis catheter, the presence of two or more central venous catheters, and the existence of organ dysfunction/failure (e.g., the need for invasive mechanical ventilation or CRRT) (Table 1). However, there were no differences in the APACHE II score and SOFA score between groups (Table 1), which might reflect similar severities of comorbid diseases. Although septic shock at the time of

Table 4 Multivariable logistic regression of factors associated with mixed-CA/B-BSIs

\begin{tabular}{llllll}
\hline Risk factors & B & S.E. & Wald & $P$ value & OR(95\% CI) \\
\hline Prior hospital stay> 7 days & 0.787 & 1.581 & 0.248 & 0.618 & $2.198(0.099,48.740)$ \\
Prior ICU stay> 2 days & 2.008 & 0.952 & 4.444 & $\mathbf{0 . 0 3 5}$ & $7.445(1.152,48.132)$ \\
Prior antibiotic exposure> 7 days & 1.289 & 1.176 & 1.203 & 0.273 & $3.631(0.362,36.383)$ \\
Prior mechanical ventilation> 2 days & -0.469 & 0.809 & 0.336 & 0.562 & $0.626(0.128,3.057)$ \\
Hemodialysis catheter & 0.707 & 0.913 & 0.600 & 0.439 & $2.028(0.339,12.133)$ \\
Two or more central venous catheters & 0.525 & 0.889 & 0.348 & 0.555 & $1.690(0.296,9.652)$ \\
Constant & -4.519 & 1.147 & 15.517 & 0.000 & $0.011(-)$ \\
\hline
\end{tabular}

Notes: Bold, indicates $P<0.05$

Abbreviations: $B$ coefficient, S.E. standard error, Wald Wald test statistic, OR odds ratio, $C I$ confidence interval, ICU intensive care unit, $C R R T$ continuous renal replacement therapy 


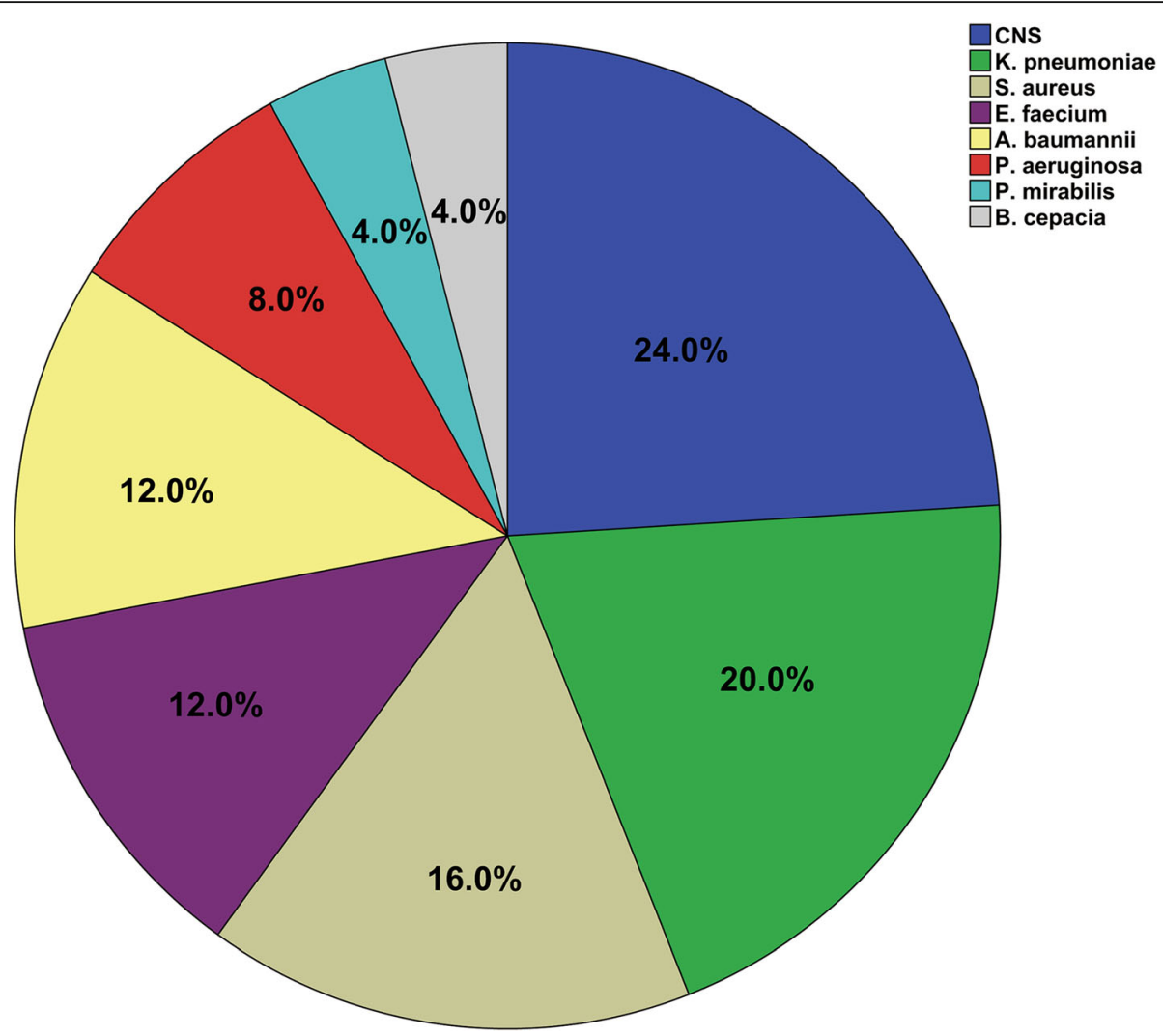

Fig. 2 Species distributions of concomitant bacteria isolated from the mixed-CA/B-BSIs

candidemia was positively associated with mixed Candida/bacterial BSIs in a previous study [5], it was not independently associated with mixed Candida/bacterial BSIs in the current study. This might be partly explained by the similar rate of appropriate antifungal therapy in both groups and a high rate of antibiotic administration (70\%) and high rate of CVC removal within $48 \mathrm{~h}$ after the first positive sample (54.2\%) in the mixed-CA/B-BSIs group (Table 2). Previous work demonstrated that more than $20.2 \%$ of nosocomial BSIs in the ICU were polymicrobial BSIs [25, 26], which is consistent with our finding that a prior ICU stay $>2$ days was an independent risk factor for mixed-CA/B-BSIs. The high incidence of polymicrobial BSI in the ICU might be explained by a suboptimal host defense altered by underlying diseases, an increased number of artificial/invasive procedures, or the application of immunosuppressive therapy in critically ill patients [26]. These results indicate that patients in the ICU are not only susceptible to BSI but also vulnerable to polymicrobial BSI, including mixedCA/B-BSIs.

In the current study, gram-positive bacteria (52\%) were the main copathogens in mixed-CA/B-BSIs, followed by gram-negative bacteria (48\%). Among all the specific

Table 5 Comparison of outcomes between mono-CA-BSI and mixed-CA/B-BSIs

\begin{tabular}{lllll}
\hline Outcomes & Total $(\boldsymbol{n}=\mathbf{1 1 7})$ & mono-CA-BSI $(\boldsymbol{n}=\mathbf{9 3})$ & mixed-CA/B-BSIs $(\boldsymbol{n}=\mathbf{2 4})$ & $\boldsymbol{P}$ value \\
\hline Total ICU stay days (IQR) & $14.0(1.0,33.0)$ & $8.0(0.0,31.5)$ & $22.0(14.3,42.2)$ & $\mathbf{0 . 0 1 0}$ \\
Total Hospitalization days (IQR) & $33.0(18.0,56.0)$ & $33.0(15.0,51.0)$ & $31.5(23.0,66.0)$ & $17.5(4.5,34.8)$ \\
Total mechanical ventilation days (IQR) & $6.0(0.0,30.5)$ & $3.0(0.0,24.5)$ & $9(37.5 \%)$ & 0.217 \\
Septic shock (n,\%) & $40(34.2 \%)$ & $31(33.3 \%)$ & $10(41.7 \%)$ & 0.019 \\
28-day mortality (n,\%) & $41(35.0 \%)$ & $31(33.3 \%)$ & $12(50.0 \%)$ & 0.701 \\
60-day mortality (n,\%) & $46(39.3 \%)$ & $34(36.6 \%)$ & $13(54.2 \%)$ & 0.229 \\
In-hospital mortality (n,\%) & $50(42.7 \%)$ & $37(39.8 \%)$ & & 0.204 \\
\hline
\end{tabular}

Notes: Bold, indicates $P<0.05$

Abbreviations: ICU intensive care unit, IQR interquartile range 


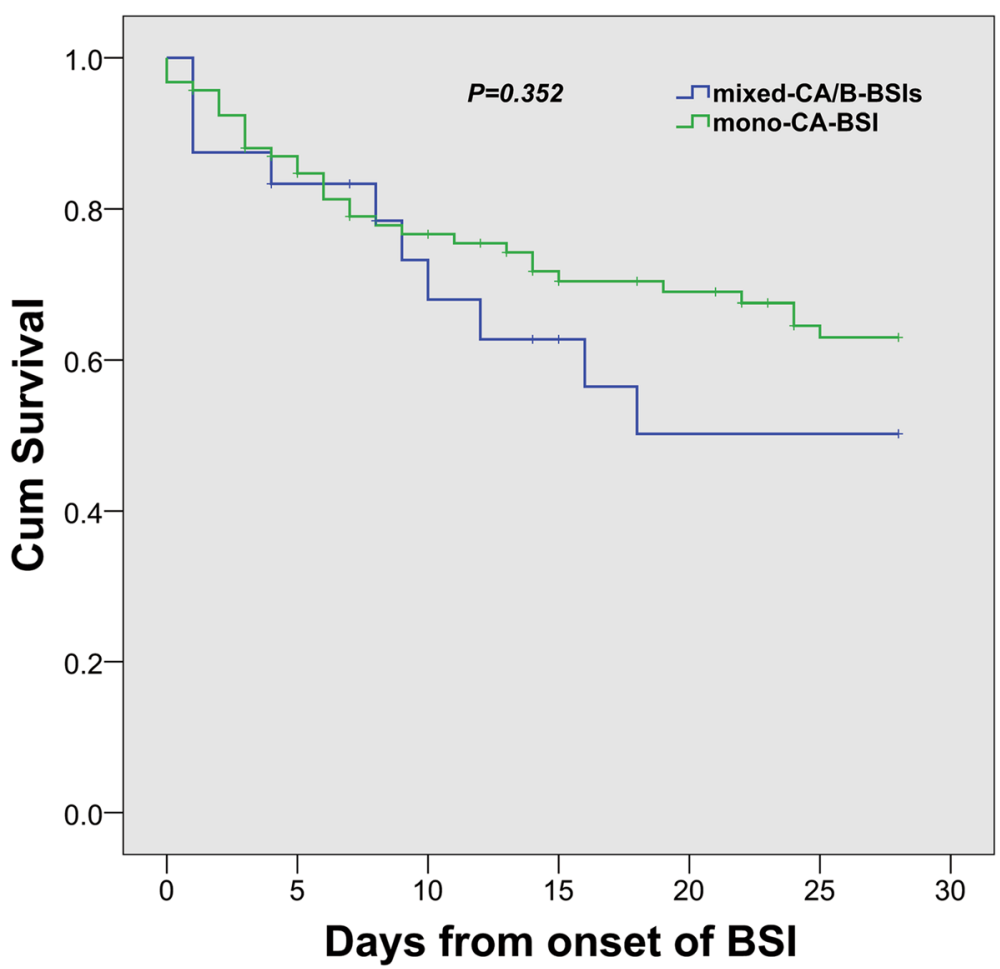

Fig. 3 Kaplan-Meier estimates of survival in patients with mixed Candida albicans/bacterial bloodstream infections and monomicrobial Candida albicans bloodstream infection

coexisting species, CNS was the predominant (24\%) species, consistent with previous reports [5]. Following $C N S$, the most prevalent copathogen species were $K$. pneumoniae (20\%) and S. aureus (16\%) (Fig. 2). This might be partly explained by the fact that the primary source of mixed-CA/B-BSIs is a CVC (29.2\%). It has been demonstrated that the polymicrobial biofilms formed by C. albicans and Staphylococcus epidermidis in vitro are commonly found in catheter-associated infection cases [27]. Although the main copathogen in Kim's study was also CNS, the second most common pathogens were Enterococcus spp. and S. aureus [5]. The gastrointestinal tract (35\%) was the most common source of mixed-CA/B-BSIs in Kim's research, while it accounted for only $5.1 \%$ of infections in the current study (Table 2). It is well known that a common source of enterococcal bacteremia is the gastrointestinal tract [28]. Consistent with a previous study that found a high proportion of $K$. pneumoniae (15.2\%) among BSIs [29], K. pneumoniae was the second most common copathogen in mixed-CA/BBSIs in our research; this might be partly due to the fact that $K$. pneumoniae has been a leading cause of HAIs over the past few decades [30]. Consistent with a previous report [6], S. aureus was the third most common isolated organism in conjunction with C. albicans in mixed-CA/BBSIs cases. S. aureus formed a substantial polymicrobial biofilm in the presence of C. albicans [31].
Although patients with mixed-CA/B-BSIs had worse outcomes (e.g., prolonged lengths of ICU stay and prolonged mechanical ventilation time) than those with mono-CA-BSI, 28 -day mortality $(41.7 \%$ vs. $33.3 \%, P=$ $0.446), 60$-day mortality $(50.0 \%$ vs. $36.6 \%, P=0.229)$ and in-hospital mortality $(54.2 \%$ vs. $39.8 \%, P=0.204)$ were similar between the two groups (Table 5, Fig. 3), similar to previous studies $[4,5,7]$. In contrast, previous studies showed polymicrobial BSI was associated with a 2.2 fold risk for increased 90-day mortality in patients with community-onset BSI [32], and also promoted an increase in 28-day mortality [33]. In our study, we found no correlation between mixed-CA/B-BSIs and mortality, which might be due to similar chronic comorbidities, a similar severity of illness at the onset of candidemia, a similar rate of fungal collection from drainage fluid, a similar delay in the initiation of empiric antifungal treatment $(75 \%$ vs. $88.2 \% P=0.697)$ and a similar rate of appropriate antifungal therapy administration $(33.3 \%$ vs. $37.6 \%, P=0.697$ ) (Table 2).

The present study has some limitations. First, this was a single-center study, and therefore, the results and conclusions might be influenced by local ecology, management practices, infection control policies, and susceptibility patterns. Second, some critical factors of mixed-CA/B-BSIs might have been missed because of the retrospective design. For example, the corticosteroid 
dosage and treatment course were not precisely obtained; thus, the immune status due to corticosteroids was unclear. We did not get information about antifungal prophylaxis and follow-up blood cultures to confirm pathogen clearance; thus, the duration of candidemia could not be obtained accurately. Third, although culture-based methods remain the gold standard to identify the causative microorganism in sepsis cases, they are notoriously insensitive, leading to challenges in implementing early interventions [34]. Nonculture diagnostic tests, such as antigen, antibody, or $\beta$-D-glucan detection assays; polymerase chain reaction (PCR) assays; and next-generation sequencing (NGS) methods, are now being performed in clinical practice as supplements to blood culture and might provide an early and/ or highly sensitive diagnosis of BSI [35-37]. Finally, although this is the first report on the risk factors for and clinical outcomes of mixed-CA/B-BSIs compared with mono-CA-BSI, the relatively small sample size may impact the confidence intervals (CIs) and analysis of risk factors. Thus, further large-scale, multicenter, prospective studies are needed.

\section{Conclusions}

Among the total CA-BSIs, mixed-CA/B-BSIs were not rare, and $C N S$ was the predominant coexisting species in mixed-CA/B-BSIs. A prior ICU stay $>2$ days was an independent risk factor for mixed-CA/B-BSIs. Although there was no difference in mortality, the prognosis of adult patients with mixed-CA/B-BSIs, including prolonged length of mechanical ventilation and prolonged length of ICU stay, was worse than that in patients with mono-CA-BSI.

\section{Abbreviations}

CA-BSI: Candida albicans bloodstream infection; mono-CABSI: Monomicrobial Candida albicans bloodstream infection; mixed-CA/BBSIs: Mixed Candida albicans/bacterial bloodstream infections; BSI: Bloodstream infection; IQR: Interquartile range; CRBSI: Catheter-related bloodstream infection; CRRT: Continuous renal replacement therapy; CVC: Central venous catheter; COPD: Chronic obstructive pulmonary disorder: SOFA: Sequential organ failure assessment; APACHE: Acute physiology and chronic health evaluation; ICU: Intensive care unit; OR: Odds ratio; Cl: Confidence interval; CNS: Coagulase-negative Staphylococcus; K. pneumoniae: Klebsiella pneumoniae; S. aureus: Staphylococcus aureus; A. baumannii: Acinetobacter baumannii; E. faecium: Enterococcus faecium; P. aeruginosa: Pseudomonas aeruginosa; B. cepacia: Burkholderia cepacia; P. mirabilis: Proteus mirabilis; S. epidermidis: Staphylococcus epidermidis; CLSI: Clinical and Laboratory Standards Institute

\section{Acknowledgements}

Not applicable.

\section{Authors' contributions}

GZ, W C and ZD designed the study, revised the manuscript and gave final approval of the version to be published; LZ, SZ and KT coordinated the writing and preparation of the manuscript and collected/analyzed the data. $F Z, C Z, K Z, J C, H Z, Y W, B T$ and ZZ collected and analyzed the data. The author(s) read and approved the final manuscript.

\section{Funding}

This manuscript was funded by the National Natural Science Foundation of China (No. 81971871, GS Zhang; No. 81772110, ZC Zhang; No. 81901941, SF Zhang) and the Natural Science Foundation of Zhejiang Province (No. LY19H150007, GS Zhang).

\section{Availability of data and materials}

We declare that the data supporting the conclusions of this article are fully described within the article, and the database is available from the first author (lizhong975717720@foxmail.com) upon reasonable request.

\section{Ethics approval and consent to participate}

This study was approved by the Human Ethics Board of the Ethics Committee of the Second Affiliated Hospital of Zhejiang University Medical College (reference number 2019-191). We ensured that the patient data remained confidential and complied with the Declaration of Helsinki. Due to its retrospective nature, the Ethics Committee determined that patient consent was not required.

\section{Consent for publication}

Not applicable.

\section{Competing interests}

The authors declare that they have no competing interests.

\section{Author details}

${ }^{1}$ Department of Critical Care Medicine, Second Affiliated Hospital, Zhejiang University School of Medicine, Hangzhou 310009, Zhejiang, China. ${ }^{2}$ Department of Critical Care Medicine, First Affiliated Hospital, Huzhou Teachers College, the First People's Hospital of Huzhou, Huzhou 313000 , Zhejiang, China. ${ }^{3}$ Department of Cardiology, Second Affiliated Hospital, Zhejiang University School of Medicine, Cardiovascular Key Laboratory of Zhejiang Province, Hangzhou 310009, Zhejiang, China. ${ }^{4}$ Department of Critical Care Medicine, Ningbo Medical Center, Li Huili Hospital, Ningbo 315040, Zhejiang, China. ${ }^{5}$ Department of Critical Care Medicine, Taizhou Municipal Hospital, Taizhou 318000, Zhejiang, China. ${ }^{6}$ Clinical Microbiology Laboratory, Second Affiliated Hospital, Zhejiang University School of Medicine, Hangzhou 310009, China.

Received: 14 June 2020 Accepted: 22 October 2020

Published online: 06 November 2020

\section{References}

1. Wisplinghoff $H$, Bischoff $T$, Tallent SM, Seifert $H$, Wenzel RP, Edmond MB. Nosocomial bloodstream infections in US hospitals: analysis of 24,179 cases from a prospective nationwide surveillance study. Clin Infect Dis. 2004;39(3): 309-17.

2. Toda M, Williams SR, Berkow EL, Farley MM, Harrison LH, Bonner L, Marceaux KM, Hollick R, Zhang AY, Schaffner W, et al. Population-based active surveillance for culture-confirmed Candidemia - four sites, United States, 2012-2016. MMWR Surveill Summ (Washington, DC : 2002). 2019; 68(8):1-15.

3. Das I, Nightingale P, Patel M, Jumaa P. Epidemiology, clinical characteristics, and outcome of candidemia: experience in a tertiary referral center in the UK. Int J Infect Dis. 2011;15(11):e759-63.

4. Bouza E, Burillo A, Munoz P, Guinea J, Marin M, Rodriguez-Creixems M. Mixed bloodstream infections involving bacteria and Candida spp. J Antimicrob Chemother. 2013;68(8):1881-8.

5. Kim SH, Yoon YK, Kim MJ, Sohn JW. Risk factors for and clinical implications of mixed Candida/bacterial bloodstream infections. Clin Microbiol Infect. 2013;19(1):62-8

6. Klotz SA, Chasin BS, Powell B, Gaur NK, Lipke PN. Polymicrobial bloodstream infections involving Candida species: analysis of patients and review of the literature. Diagn Microbiol Infect Dis. 2007;59(4):401-6.

7. Pulimood S, Ganesan L, Alangaden G, Chandrasekar P. Polymicrobial candidemia. Diagn Microbiol Infect Dis. 2002;44(4):353-7.

8. Chen XC, Xu J, Wu DP. Clinical characteristics and implications of mixed candida/bacterial bloodstream infections in patients with hematological diseases. Eur J Clin Microbiol Infect Dis. 2020;39(8):1445-52.

9. Antoniadou A, Torres HA, Lewis RE, Thornby J, Bodey GP, Tarrand JP, Han $\mathrm{XY}$, Rolston KV, Safdar A, Raad II, et al. Candidemia in a tertiary care cancer 
center: in vitro susceptibility and its association with outcome of initia antifungal therapy. Medicine. 2003;82(5):309-21.

10. Almirante B, Rodriguez D, Park BJ, Cuenca-Estrella M, Planes AM, Almela M, Mensa J, Sanchez F, Ayats J, Gimenez M, et al. Epidemiology and predictors of mortality in cases of Candida bloodstream infection: results from population-based surveillance, Barcelona, Spain, from 2002 to 2003. J Clin Microbiol. 2005;43(4):1829-35.

11. Rajendran R, Sherry L, Nile CJ, Sherriff A, Johnson EM, Hanson MF, Williams C, Munro CA, Jones BJ, Ramage G. Biofilm formation is a risk factor for mortality in patients with Candida albicans bloodstream infection-Scotland, 2012-2013. Clin Microbiol Infect. 2016;22(1):87-93.

12. CDC. Bloodstream infection event (central line-associated bloodstream infection and non-central line-associated bloodstream infection). Atlanta: CDC; 2015.

13. CDC. Identifying Healthcare-associated Infections (HAI) for NHSN Surveillance. Atlanta: CDC; 2015.

14. Institute CaLS. Reference method for broth dilution antifungal susceptibility testing of yeasts, third informational supplement. Wayne: M27-A3; 2008.

15. Institute CaLS. Performance standards for antimicrobial susceptibility testing. 28th ed. Wayne: supplement M100; 2018.

16. Ascioglu S, Rex JH, de Pauw B, Bennett JE, Bille J, Crokaert F, Denning DW Donnelly JP, Edwards JE, Erjavec Z, et al. Defining opportunistic invasive fungal infections in immunocompromised patients with cancer and hematopoietic stem cell transplants: an international consensus. Clin Infect Dis. 2002;34(1):7-14

17. Mermel LA, Allon M, Bouza E, Craven DE, Flynn P, O'Grady NP, Raad II, Rijnders BJ, Sherertz RJ, Warren DK. Clinical practice guidelines for the diagnosis and management of intravascular catheter-related infection: 2009 update by the Infectious Diseases Society of America. Clin Infect Dis. 2009; 49(1):1-45.

18. Morrell M, Fraser VJ, Kollef MH. Delaying the empiric treatment of candida bloodstream infection until positive blood culture results are obtained: a potential risk factor for hospital mortality. Antimicrob Agents Chemother. 2005:49(9):3640-5.

19. Pappas PG, Kauffman CA, Andes D, Benjamin DK Jr, Calandra TF, Edwards JE Jr, Filler SG, Fisher JF, Kullberg BJ, Ostrosky-Zeichner L, et al. Clinical practice guidelines for the management of candidiasis: 2009 update by the Infectious Diseases Society of America. Clin Infect Dis. 2009;48(5):503-35.

20. Zasowski EJ, Claeys KC, Lagnf AM, Davis SL, Rybak MJ. Time is of the essence: the impact of delayed antibiotic therapy on patient outcomes in hospital-onset Enterococcal bloodstream infections. Clin Infect Dis. 2016; 62(10):1242-50.

21. Singer M, Deutschman CS, Seymour CW, Shankar-Hari M, Annane D, Bauer M, Bellomo R, Bernard GR, Chiche JD, Coopersmith CM, et al. The third international consensus definitions for Sepsis and septic shock (Sepsis-3). Jama. 2016;315(8):801-10.

22. Wang YC, Ku WW, Yang YS, Kao CC, Kang FY, Kuo SC, Chiu CH, Chen TL Wang FD, Lee AY. Is Polymicrobial Bacteremia an Independent Risk Factor for Mortality in Acinetobacter baumannii Bacteremia? J Clin Med. 2020;9(1).

23. Liu Q, Wu J, Wang Z, Wu X, Wang G, Ren J. Polymicrobial bacteremia involving Klebsiella pneumoniae in patients with complicated intraabdominal infections: frequency, co-pathogens, risk factors, and clinical outcomes. Surg Infect. 2019;20(4):317-25.

24. Zheng C, Cai J, Liu H, Zhang S, Zhong L, Xuan N, Zhou H, Zhang K, Wang Y, Zhang $X$, et al. Clinical characteristics and risk factors in mixed-Enterococcal bloodstream infections. Infect Drug Resist. 2019;12:3397-407.

25. Sancho S, Artero A, Zaragoza R, Camarena JJ, Gonzalez R, Nogueira JM Impact of nosocomial polymicrobial bloodstream infections on the outcome in critically ill patients. Eur J Clin Microbiol Infect Dis. 2012;31(8): 1791-6.

26. Rello J, Quintana E, Mirelis B, Gurgui M, Net A, Prats G. Polymicrobial bacteremia in critically ill patients. Intensive Care Med. 1993;19(1):22-5.

27. Adam B, Baillie GS, Douglas LJ. Mixed species biofilms of Candida albicans and Staphylococcus epidermidis. J Med Microbiol. 2002;51(4):344-9.

28. Billington EO, Phang SH, Gregson DB, Pitout JD, Ross T, Church DL, Laupland KB, Parkins MD. Incidence, risk factors, and outcomes for Enterococcus spp. blood stream infections: a population-based study. Int $J$ Infect Dis. 2014:26:76-82

29. Chen S, Liu S, Yuan X, Mai H, Lin J, Wen F. Etiology, drug sensitivity profiles and clinical outcome of bloodstream infections: a retrospective study of 784 pediatric patients with hematological and neoplastic diseases. Pediatr Hematol Oncol. 2019:36(8):482-93.

30. Moradigaravand D, Martin V, Peacock SJ, Parkhill J. Evolution and Epidemiology of Multidrug-Resistant Klebsiella pneumoniae in the United Kingdom and Ireland. mBio. 2017;8(1).

31. Harriott MM, Noverr MC. Candida albicans and Staphylococcus aureus form polymicrobial biofilms: effects on antimicrobial resistance. Antimicrob Agents Chemother. 2009;53(9):3914-22

32. Yo CH, Hsein YC, Wu YL, Hsu WT, Ma MH, Tsai CH, Chen SC, Lee CC. Clinical predictors and outcome impact of community-onset polymicrobial bloodstream infection. Int J Antimicrob Agents. 2019.

33. Pavlaki M, Poulakou G, Drimousis P, Adamis G, Apostolidou E, Gatselis NK, Kritselis I, Mega A, Mylona V, Papatsoris A, et al. Polymicrobial bloodstream infections: epidemiology and impact on mortality. J Glob Antimicrob Resist. 2013;1(4):207-12.

34. Pappas PG, Kauffman CA, Andes DR, Clancy CJ, Marr KA, Ostrosky-Zeichner L, Reboli AC, Schuster MG, Vazquez JA, Walsh TJ, et al. Clinical practice guideline for the Management of Candidiasis: 2016 update by the Infectious Diseases Society of America. Clin Infect Dis. 2016;62(4):e1-50.

35. Chibabhai $\vee$, Fadana V, Bosman N, Nana T. Comparative sensitivity of 1,3 beta-D-glucan for common causes of candidaemia in South Africa. Mycoses. 2019;62(11):1023-8.

36. McKeating C, White PL, Posso R, Palmer M, Johnson E, McMullan R. Diagnostic accuracy of fungal PCR and $\beta$-d-glucan for detection of candidaemia: a preliminary evaluation. J Clin Pathol. 2018;71(5):420-4.

37. Chiu CY, Miller SA. Clinical metagenomics. Nat Rev Genet. 2019;20(6):341-55.

\section{Publisher's Note}

Springer Nature remains neutral with regard to jurisdictional claims in published maps and institutional affiliations.

Ready to submit your research? Choose BMC and benefit from:

- fast, convenient online submission

- thorough peer review by experienced researchers in your field

- rapid publication on acceptance

- support for research data, including large and complex data types

- gold Open Access which fosters wider collaboration and increased citations

- maximum visibility for your research: over $100 \mathrm{M}$ website views per year

At $\mathrm{BMC}$, research is always in progress.

Learn more biomedcentral.com/submissions 